\title{
Exploiting Human Cooperation in Human-Centered Robot Navigation
}

\author{
Thibault Kruse*, Alexandra Kirsch*, E. Akin Sisbot ${ }^{\dagger}$, Rachid Alami $^{\dagger}$
}

\begin{abstract}
Robot path planning has traditionally concentrated on collision-free paths. For robots that collaborate closely with humans, however, the situation is different in two respects: 1) the humans in the robot's environment are not randomly moving objects, but cognitive beings who can deliberately make way for a robot to pass and 2) the quality of a navigation plan depends less on quantitative efficiency criteria, but rather on the acceptance of humans. In this paper, we introduce a robot navigation approach that takes into account human-centered requirements and the collaborative nature of the interaction between the human and the robot.
\end{abstract}

\section{Motivation}

An autonomous robot that is not just acting for humans, but collaborates closely with people, must consider human comfort and social acceptability in all its actions, including its navigation behavior. A prerequisite of human comfort is legible behavior, which means that an ordinary, uninstructed person can understand and anticipate the robot's actions.

Robot motion planners usually try to find provably collision-free paths, without considering moving objects or comfort of humans. While this yields correct plans with regards to safe behavior, we think that it leads to overall behavior that is less efficient and less legible than it could be if the robot assumed a collaborating human partner to be benevolent and to support the robot in its actions. When several humans operate in constrained space, they don't just avoid each other, but coordinate their movements e.g. by stepping aside if another person needs to pass. In this paper, we describe how a robot could exploit the fact that people are frequently moving in household tasks and that people might make way for a robot to pass in order to achieve more legible and acceptable robot behavior. As an instance of a robot that collaborates with humans, we consider a household assistant that helps elderly or disabled people in their daily chores (in an environment as shown in Figure 1). In such a context, we assume that people are mostly moving, for example between the table and the sink in a kitchen, fetch things from the refrigerator or go into another room.

In confined spaces, a safe plan, which avoids the human at all costs, can be unnatural. In the situation shown in Figure 2, the direct way is blocked by persons. The alternative path using the free space is extremely long. With the assumption of the persons being cooperative, the robot would show a more natural behavior when trying to pass by making the persons move.

* IAS, TU München Boltzmann Str. 3, 85748 Garching, Germany. Email: \{kruset,kirsch\}@in.tum.de

† CNRS; LAAS; 7 avenue du Colonel Roche, F-31077 Toulouse, France. Université de Toulouse; UPS, INSA, INP, ISAE; LAAS; F-31077 Toulouse, France. Email: \{rachid.alami,easisbot\}@laas.fr

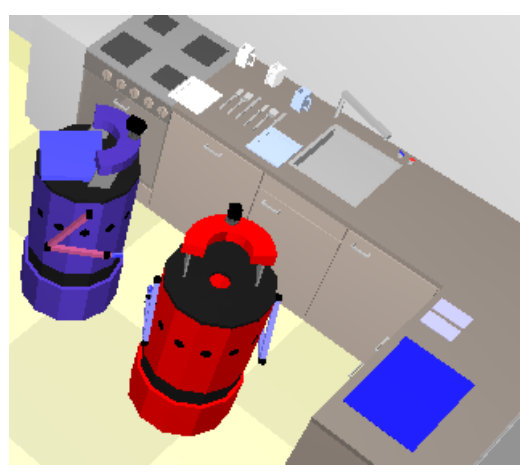

Fig. 1: Example spatial conflict for two agents in the Gazebo simulator trying to reach a position to grasp items

In this paper, we present several methods to generate more efficient plans under the assumption that a human might move out of the way and strategies for plan execution to account for human movement while still assuring safety.

Finally, we didn't use explicit communication between the agents. For navigation in indoor contexts, humans rarely need to communicate verbally. A constantly communicating robot would certainly violate social rules. Besides, communicating a navigation plan on a level that is understandable by a human, is not trivial. We will point out methods where additional communication might clarify a situation, but we think it should be avoided if possible.

The contributions of this paper are

- an extension of the human-aware navigation planner HANP to make plans more efficient in the presence of moving humans;

- means for plan execution, which ensure safety and take into account possible future movement of humans;

- an experimental evaluation of the extended planning and execution mechanisms.

The next section provides an overview of related work. The following one introduces the human-aware navigation planner, which we use as a basis for our cooperative navigation planning. After that we describe our extensions to motion plan generation and plan execution. The following evaluation compares different combinations of these strategies in different environments and discusses their applicability. Finally we present a conclusion.

\section{RELATED WORK}

Alami et al. [1] present a plan merging paradigm for multi-robot scenarios. Even though this method solves robot coordination problems and blocking situations, it relies on explicit symbolic communication between robots about motion plans, which is not applicable to HRI. 


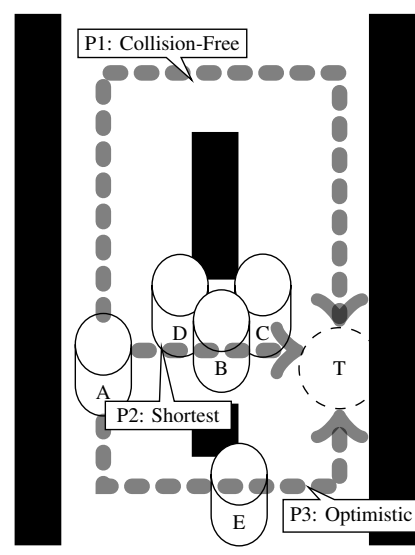

Fig. 2: Strategy alternatives: P1-Collision free path, P2shortest path ignoring humans, P3-optimistic path balancing length vs. human comfort. This shows optimistic planning being different from path planning where humans are simply ignored.

Philippsen [8] gives a comprehensive overview of other motion planning techniques, which however also do not take into account human comfort.

Recently, Hansen et al. [5] provided a solution for positioning a robot in relation to a human according to the situation classified by motion pattern analysis.

Foka and Trahanias [4] have presented a method for prediction of human motion using a partially observable Markov decision process (POMDP). A similar work on prediction of pedestrians is given in [11].

Müller et al. [7] implemented an iterative $A^{*}$ algorithm for a robot preferring to follow moving humans rather than moving around them. This was an effort to imitate one aspect of human motion behavior.

Tadokoro et al. examined robot motion planning in presence of moving humans in [10]. In this paper they used an occupancy grid which does not solve the problem of confined spaces. They equally used simulation to evaluate their approach.

The related work on robot motion planning can be classified into those approaches focusing on a geometric solution and dynamic environments, and those focusing on producing comfortable and legible robot movement for present humans. Ours belongs to the second category, as we target legibility and acceptance of robot motion.

\section{Human Aware Navigation}

The presented work on cooperative motion planning uses the human-aware navigation planner (HANP) to generate socially acceptable plans under the assumption of a static human [9]. By relaxing some constraints in HANP and changing some cost functions, we could achieve cooperative behavior that is more suitable for moving humans.

HANP generates a path for a robot to move in the presence of humans. Unlike other path planners, it considers additional constraints during motion planning based on user studies on human-robot space sharing $[2,6]$ in the form of a

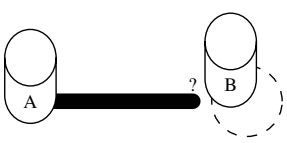

(a) Endpoint blocked

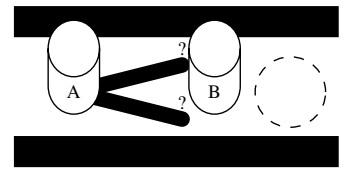

(b) Corridor middle conflict
Fig. 3: Cases where static planning fails due to agent B blocking collision free solutions for Agent A to goal.

sophisticated cost function, that increases costs of free space in the environment based on how much discomfort a robot imposes on present humans. HANP uses $\mathrm{A}^{*}$ search, and the minimal path to a goal is a compromise between minimal path length and minimal human discomfort. This approach has been validated in different simulated domains with a varying number of humans and different pieces of furniture and obstacles.

HANP in its current state computes paths in static environments, which means the planner considers the humans as fixed. When planning on a map, we can compare the least cost path HANP finds in a map given with a human compared to the human not being present. HANP can then return:

1) A path which is optimal even if the humans moved

2) No path, although a path is possible should humans move

3) A path which is much worse than a path possible should humans move

4) No path, and no path is possible even if humans moved

The first case and fourth case cannot be improved. We focus on the second and third case, where the dynamics allow solutions which a static planner does not consider.

Figure 3 illustrates examples for the second class of results: the goal position is currently blocked by another agent in (a). And in (b) an agent is in the way in a room too narrow to plan a safe path around it. These are cases where humans wouldn't capitulate, but coordinate their actions with the other person, in most cases implicitly without the need to communicate verbally.

The third case - of finding a very inefficient plan - is illustrated in Figure 2. Although HANP would find a valid plan (the long way around the large obstacle) such a plan cannot be considered most legible or efficient. A human in the same situation would rather indicate its intended goal and hope for other humans to make way rather than moving the long way around. The figure also shows that when there are several paths blocked by humans, some paths are preferable based on the number and activities of the humans.

We expect the second and third case to happen frequently in realistic domestic environments, and we expect that robot behavior failing in those cases to be quite unsatisfactory. Next we describe methods for generating plans in HANP, so that the robot shows adequate behavior in such situations.

\section{Cooperative Planning and Plan Execution}

We propose a change to HANP by changing the search space and cost functions to account for potential dynamics of the environment. We further introduce an execution 
framework for dynamic navigation plans, which takes into account the additional safety constraints which arise by the modified costs used by HANP and considers possible future movement of humans. The cost functions described in [9] have been slightly modified mostly to improve the robot's behavior when in very close proximity to the human. We used the following cost functions, with dis being the distance of a point to the human, and ang the absolute angle between the xy-orientation of the human and the vector from the human to the point:

For safety: A sigmoid function of the euclidean distance of a point to a human in $\mathrm{x}$ and $\mathrm{y}$ direction.

$$
c_{\text {dist }}(\text { dis })= \begin{cases}\left.\left(\frac{\cos \left(\frac{d i}{6} \times \frac{\pi}{2}\right)}{c+d i s}\right)\right)^{a}, & \text { if dis }<b \\ 0, & \text { else }\end{cases}
$$

Parameter $a$ changes the gradient of the function. $b$ and $c$ the radius and the inflection circle radius. After several tries we settled for $(a=3, b=1.3 m, c=0.6 m)$ for standing humans, the same except $b=1.5 \mathrm{~m}$ for moving humans.

For visibility: Increases costs for the robot to approach a static human outside their sight.

$$
\begin{aligned}
& c_{v i s}(\text { dis }, \text { ang })= \\
& \begin{cases}\cos \left(\frac{d i s}{e} \times\right. & \left.\frac{\pi}{2}\right) \times\left(f+g \times\left(a n g-45^{\circ}\right)\right), \\
& \text { if dis }<e \text { and } \text { ang }>45^{\circ} \\
0, & \text { else }\end{cases}
\end{aligned}
$$

Parameter $e$ limits the radius of the function while $f$ changes the gradient towards the human and $g$ the gradient towards his back. We use values ( $e=2 \mathrm{~m}, f=40, g=50), g=40$ for moving humans.

As prediction: Increases costs in front of a moving human. For static humans, the function is always 0 .

$$
c_{\text {pred }}(\text { dis, ang })=
$$

$$
\begin{cases}\cos \left(\frac{d i s}{e} \times\right. & \left.\frac{\pi}{2}\right) \times\left(f+h \times\left(180^{\circ}-\text { ang }\right)\right), \\ & \text { if dis }<e \text { and ang }<45^{\circ} \\ 0, & \text { else }\end{cases}
$$

Parameters $e$ and $e$ are the same as for visibility, $h$ changes the gradient in front of the human.

For hidden zones: Evaluates in original HANP the space behind large objects which is not visible for a person. This cost function was not used in our experiments, as we had no corresponding situation.

With these cost functions, an $\mathrm{A}^{*}$ search is performed, with the path-cost function denoted $g(x)$ in the literature constructed as follows:

$$
\begin{aligned}
& g\left(x_{0}\right)=0 \\
& g\left(x_{i+1}\right)=g\left(x_{i}\right)+\alpha \times\left|\overline{x_{i} x_{i+1}}\right|+ \\
& \beta \times c_{\text {dist }}+\delta \times c_{v i s}+\gamma \times c_{p r e d} .
\end{aligned}
$$

Parameters $\alpha$ to $\gamma$ regulate the priorities of the individual cost functions and path length. We used $(\alpha=40, \beta=8, \gamma=$ $\delta=1)$. As admissible $\mathrm{A}^{*}$ heuristic function we used $h(x)=$ $\alpha \times\left|\overline{x_{i} g o a l}\right|$. For more than one human in the environment, the functions in HANP are combined taking the maximum value of the cost functions for each human.

\section{A. Predictive Planning}

The cost function $c_{\text {pred }}$ is an addition to HANP that increases costs in front of a moving human, such that a robot will attempt to avoid this area. These new costs are

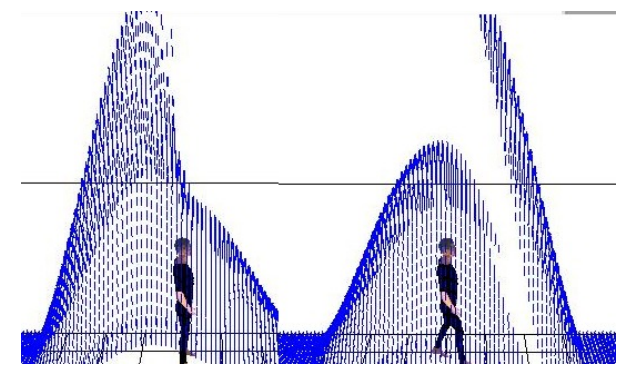

Fig. 4: Costs in front of moving human increased in comparison to standing human. For a standing human, we avoid moving behind the human by assigning high costs to that area. For moving humans, we rather avoid moving in their direction of motion.

illustrated in Figure 4 This is a heuristic assuming humans always move forward, which may easily be changed to use more sophisticated predictions of human motion.

Predictive planning uses heuristics that may fail, meaning the human may not move in the predicted ways. Our controller therefore also replans when the human stops after a period of motion, changing the cost functions for the now static human.

\section{B. Optimistic Planning}

As described in Section III, HANP uses several cost functions for modeling social aspects of the navigation problem. In the standard costs of HANP, an area around the human is removed from search where the robot would collide with the human. This prevents the robot under all circumstances to hit the human and thus guarantees safety. We relax this safety requirement slightly and shift it to the plan execution. For humans or other agents in the environment we use all cost functions already present in HANP, removing just the collision checks. The other human-aware cost functions already induced high costs in areas of potential collision.

Using these cost functions ensures that the robot will use a path around the human if the detour is not too big. However, when an alternative path produces higher costs (e.g. by a longer path or the violation of other constraints like visibility), HANP produces a navigation path intersecting with the human position, thus possibly leading to a blocking. Only when the human moves out of the way, the robot can follow this path and achieve its goal.

The modified planner can yield one of the these results:

1) A free path

2) A path which requires other agents to move.

3) A path requiring other agents to move where it is impossible for them to move

4) No path and no path is possible even if other agents moved.

In the cases shown in Figure 2 and Figure 3, optimistic planning will return solutions which require the other agent to move. It might be surprising that the third class of results may find a path which cannot work in any case. We could not prevent this due to the complexity of the search space particularly for a situation of $n$ agents. If that 


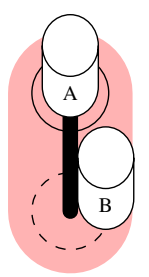

(a)

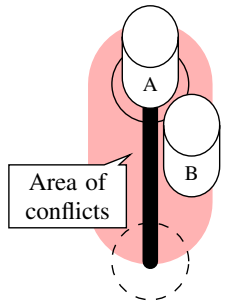

(b)

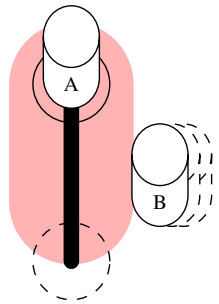

(c)
Fig. 5: Possible conflict situations during motion. (a) Goal Conflict (b) Path conflict (c) Potential conflict The red region shows the area of conflict, the black line shows the planned path for robot $\mathrm{A}$.

happens, or other agents refuse to move, after a timeout the robot continues to search considering those agents as static obstacles.

\section{Dynamic Plan Execution}

The modifications in the use of HANP can lead to collision-prone paths. For executing the plans, we define possible conflict situations during plan execution with the concept of area of conflict. In Figure 5 the gray areas illustrate the area of conflict, which is defined as the area in the direction of the robot movement, covering a certain range, in which the robot can be expected to stop if necessary.

The length of the area of conflict needs to be defined according to how quickly the robot can stop and what distance he should have from humans then. For our experiment we used a local segment of the path which would move the robot maximally once his diameter from his current position, allowing him to stop at a distance of $30 \mathrm{~cm}$ to the bounding circle of a human.

Figures 5a and 5b show factual conflicts, which would cause the robot to be blocked in its approach to a goal. We distinguish between situations where the other agent is on the target position and where it is not. The difference is that in the latter case, another path around the agent might still be a viable alternative for conflict resolution. We assume optimistically that if the other agent cooperates, moving along this path will be more efficient than choosing a different path. In the case of another agent blocking the target point, possible resolution strategies are to wait for the other agent to move, to initiate communication to demand the agent to move, or to plan to a different point.

The robot can also expect future conflicts by observing agents that are not in a state of conflict at present, but can be assumed to enter this state very soon (considering their motion) as shown in Figure 5c. A controller may as a heuristic for conflict avoidance drop the speed or even stand and wait until the other agent either stands or moves away from the area of conflict.

The navigation task can fail if the robot's goal position keeps being blocked by another agent or there is no alternative path if the human doesn't step aside. So far the controller fails in this case. In future work a higher level controller could decide to start communication with the person or change its course of action completely.

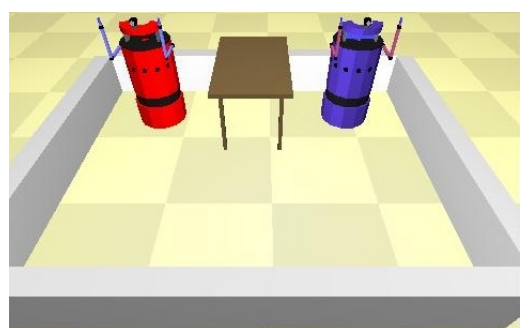

Fig. 6: Experimental setup: The robots have a diameter of $0.6 \mathrm{~m}$, the task was to go to the opposite corner of the room as shown in Figure 8.

\section{Evaluation OF STRATEGIES}

We evaluated two variants of the base motion controller in an environment frequently generating conflict situations. In addition, to demonstrate increased legibility of robot motion with optimistic planning, we compared the behavior for two robots in a small kitchen environment.

\section{A. Experimental setup}

We analyzed the properties of our algorithms in a simulation with two robots, using a custom Player driver for the motion control, HANP with Genom[3] for the path planning, and Gazebo for the physical simulation of the 3D environment which models robot motion very realistically. By using the simulator we can validate several properties at lower costs than in user studies, which is particularly important for robot behavior in dynamic environments, as the space of possible situations to be checked is much larger than for static cases. The HANP algorithm takes 500ms on average to find a solution, so each robot could replan once per second. We evaluated dynamic situations in which both robots moved to individual goals, with the shortest paths crossing each other. The environments we examined are illustrated in Figure 6. The strategies we compared are

A: Optimistic planning,

B: Optimistic planning with predictive planning.

C: Non-Optimistic planning

In B we assessed predictive planning, where robots will stop and wait when the other is close to their area of conflict and moving, as shown in Figure 5c. Natural dithering prevents that strategy to cause a livelock, meaning the first robot to move again will remain moving with that strategy.

In the situation in Figure 6, the direct paths of the robots cross each other. This is like a crossroads scenario, only that in this form it is more likely to occur in a household. We measured the time needed for both robots to reach their goal positions, the time the robots stood waiting, and the total distance traveled.

As a separate experiment, we were interested in the effects of using the optimistic and predictive planning algorithm in a less artificial situation. We set up a kitchen scenario in simulation, as can be seen in Figure 9. We let two autonomous independent robots, representing a robot $\mathrm{R}$ and a human $\mathrm{H}$, execute two different independent plans at the same time. The robots had to bring items from a sink to a table, the plans were deliberately chosen so that robot $\mathrm{R}$ 


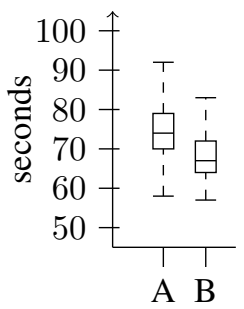

(a) Time to goal

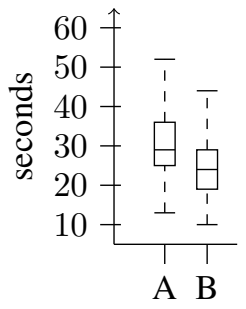

(b) Stopped time

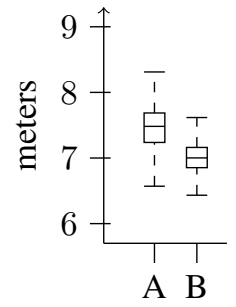

(c) Path length
Fig. 7: Quantitative results. A and B both used optimistic planning. A: Non-predictive planning, B: predictive planning. Results for room with table for 300 successful samples. Strategy C never succeeded. Values are the sums for both robots in each trial.

would in three occasions have to move to a spot robot $\mathrm{H}$ occupied temporarily. We performed two sets of 30 trials. We always had the human representing robot $\mathrm{H}$ planning the path optimistically and predictive, as in strategy B. R had a motion planner that was neither optimistic nor predictive in one set of experiments, and an optimistic predictive planner in the other set. The robot using the non-optimistic motion planner used a strategy of waiting until a spot became free without ever failing.

\section{B. Results}

Figure 7 shows the quantitative results for the first experiment. No results are given for strategy $\mathrm{C}$, as the robots would always end in a deadlock. The figure shows boxplots, the box indicating the range where $50 \%$ of all values occurred, the line in the box being median, and the "whiskers" representing the minimum and maximum values.

We analyzed the time until both robots reached their goal position ("Time to goal"), the sum of the times the robot waited during their navigation task ("Stopped time") and the distance both robots covered during one trial ("Path length").

The results first of all show that optimistic planning is a suitable solution for confined spaces, returning path solutions where the path is blocked, yet becomes available after a change in the world by other agents moving.

The differences in performance between experiments $\mathrm{A}$ and $\mathrm{B}$ can be explained due to swaying behavior which naturally occurs with non-predictive planners. Figure 8 shows samples of trajectories traversed during the experiments. In the depicted non-predictive case (a), the robot coming from the right realizes very late the potential conflict with the other robot and has to change its path. Using predictive planning, this visible swaying is greatly reduced, because both robots adapt their paths at an earlier point in time.

The kitchen experiment on the one hand validated that for realistic scenarios, shifting the safety requirement from planning to control did not make the robot behave less safe. More importantly for us however, is the effect on the legibility of the robot's behavior. As Figure 9 shows, optimistic planning made a visible difference to robot R. In the case of non-optimistic planning, robot $\mathrm{R}$ merely waited at the position it was in when the goal region became blocked.

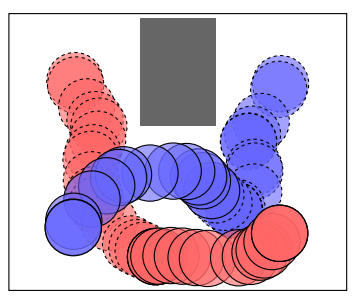

(a) Non-predictive planner

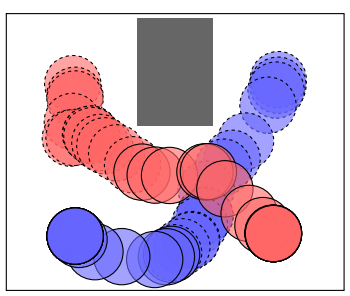

(b) Predictive planner
Fig. 8: Behavior in a room with a table. The circles represent the bounding circles of two robots at different times. Earlier spots have dashed borders. With static planning, both robots first plan to take the outer "lane", making the blue robot (coming from the right) sway.

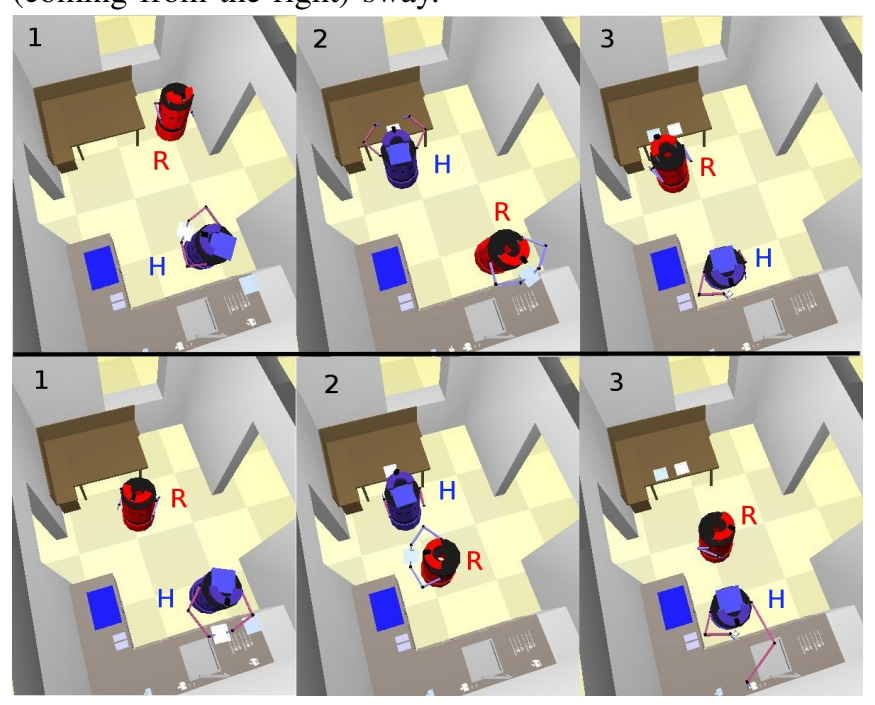

Fig. 9: Snapshots from the kitchen experiment. Two individual trials, from left to right. The top one shows robot $\mathrm{R}$ with non-optimistic strategy, the bottom one with both robots using optimistic and predictive strategy. The images show instants where robot R needed to go to the spot of "human" H next.

From looking at the pictures the intention of $\mathrm{R}$ in the first row of pictures cannot be guessed. Whereas using optimistic planning, robot $\mathrm{R}$ waited closer to its destination in all three cases, conveying legibly its intend to move there. This behavior also improves performance of plans, as necessary waiting times are used to travel some of the distance, though we are focused on improvement of the human experience.

\section{Discussion and Future Work}

Our evaluation has shown that our proposed measures for collaborative, human-centered navigation leads to more efficient and more importantly to more legible plans than using the assumption of humans as mere obstacles. The evaluation was performed in simulations with two robots. Efficiency gains were due to reduction of swaying, better usage of necessary waiting times, and also planning alternative routes exploiting motion of other agents. We showed improvements due to the first two causes. We assume the gain due to agent cooperation, granted in theory, can best be shown in trials with humans, which we intend to do as future work. Considering the work of Müller et al. [7] who made robots 
move without considering human comfort and expecting the human to move away, we expect that the navigation will be more smooth as people will probably make way for a robot or stay clearly on their intended path.

Optimistic planning offers most benefits in situations where the human blocking the way is at least several steps away from the robot, such that he/she cannot guess the intentions of the robot. These are the situations where a robot approaching on a blocked path optimistically both exploits the fact that the human might move for an arbitrary reason, as well as expressing its own intentions implicitly. The first experiment shows that in some geometric cases for multirobot scenarios, deadlocks of non-optimistic methods can heuristically be avoided, without detecting them first.

An open question is how the robot can disambiguate if it is approaching a human for interaction or trying to pass through. This would require sophisticated reasoning about the overall situation. In this case some explicit form of communication might be necessary.

We are aware that optimistic planning may trap humans in passages too small for two agents to pass and thus causing discomfort to the human. One approach could be a further adaptation of the cost function in HANP to provide costs indicating the human's possibilities of moving away from the current position, e.g. measuring "crowdedness" of areas.

To reduce the resource consumption of constant replanning on a robot when humans move, methods to evaluate the need for replanning could also be investigated.

\section{Conclusion}

Generating and executing navigation plans for collaboration with humans imposes additional challenges on a robot controller with respect to social rules and legibility. The generation and execution of such plans differs in domains with relatively static humans (for example when people are sitting or talking to each other as at a cocktail party) and those with moving humans (for example when performing household tasks).

In this paper, we present measures to use the existing human-aware navigation planner HANP in highly dynamic, collaborative situations. By introducing the notion of optimistic planning, HANP finds more and potentially more acceptable solutions to robot motion in the presence of humans. Predictive planning adds specific costs to account for the predicted movement of a human at planning time.

Using these measures, the generated plans are not guaranteed to be collision-free, but leave it to the motion controller to create safe behavior by waiting in front of other agents. Our plan execution is based on the concept of conflicts defined by the area of conflict along the robot's planned path. In conflicting situations, the robot stops and has several choices of how to proceed: replan at once, wait for the human to move or possibly to communicate with the person.

The empirical evaluation using two robots that act independently - one in the role of the human, the other as the robot - shows an improvement of the robot behavior considering the legibility of its movement and indicates a possible efficiency gain. Beyond the robot-robot experiments we have conducted, we expect even more considerable results when using the approach in a human-robot setting where humans can be expected to interpret the robot's intentions and thus produce more legibility as the robot can stay better on its intended path.

Acknowledgements This work was supported by the CoTeSys (Cognition for Technical Systems) cluster of excellence, the BFHZ and by the European Community's Information and Communication Technologies Seventh Framework Programme [FP7/2007-2013] under grant agreement n [215805], the CHRIS project

\section{REFERENCES}

[1] R. Alami, S. Fleury, M. Herb, F. Ingrand, and F. Robert. Multi robot cooperation in the Martha project. IEEE Robotics and Automation Magazine, 5(1), 1998.

[2] K. Dautenhahn, M. Walters, S. Woods, K. L. Koay, C. L. Nehaniv, E. A. Sisbot, R. Alami, and T. Siméon. How may i serve you?: a robot companion approaching a seated person in a helping context. In $A C M$ SIGCHI/SIGART International Conference on HumanRobot Interaction, HRI, pages 172-179, 2006.

[3] S. Fleury, M. Herrb, and R. Chatila. Genom: A tool for the specification and the implementation of operating modules in a distributed robot architecture. In IROS, pages 842-848, 1997.

[4] A. Foka and P. Trahanias. Predictive autonomous robot navigation, 2002.

[5] S. T. Hansen, M. Svenstrup, H. J. Andersen, and T. Bak. Adaptive human aware navigation based on motion pattern analysis. In Robot and Human Interactive Communication, 2009., Toyama, Japan, Sept.-Oct. 2009.

[6] K. Koay, E. Sisbot, D. Syrdal, M. Walters, K. Dautenhahn, R. Alami. Exploratory studies of a robot approaching a person in the context of handing over an object. In AAAI - Spring Symposium 2007: Multidisciplinary Collaboration for Socially Assistive Robotics.

[7] J. Müller, C. Stachniss, K. O. Arras, and W. Burgard. Socially inspired motion planning for mobile robots in populated environments. In International Conference on Cognitive Systems (CogSys'08), Karlsruhe, Germany, 2008.

[8] R. Philippsen. Motion Planning and Obstacle Avoidance for Mobile Robots in Highly Cluttered Dynamic Environments. PhD thesis, Ecole Polytechnique Fédérale de Lausanne, 2004.

[9] E. A. Sisbot, L. F. Marin-Urias, R. Alami, T. Simeon. A human aware mobile robot motion planner. IEEE Transactions on Robotics, 23:874-883, 2007.

[10] S. Tadokoro, M. Hayashi, Y. Manabe, Y. Nakami, and T. Takamori. On motion planning of mobile robots which coexist and cooperate with human. IROS, IEEE/RSJ, 2:2518, 1995.

[11] B. Ziebart, N. Ratliff, G. Gallagher, C. Mertz, K. Peterson, J. A. D. Bagnell, M. Hebert, A. Dey, and S. Srinivasa. Planning-based prediction for pedestrians. In Proc. IROS 2009, October 2009. 